\title{
A new approach for solving multi-pantograph type delay differential equations
}

\author{
Nidal Anakira', Ali Jameel ${ }^{2}$, Mohmmad Hijazi ${ }^{3}$, Abedel-Karrem Alomari ${ }^{4}$, Noraziah Man ${ }^{2}$ \\ ${ }^{1}$ Department of Mathematics, Faculty of Science and Information Technology, Irbid National University, Irbid, Jordan \\ ${ }^{2}$ School of Quantitative Sciences, Universiti Utara Malaysia (UUM), Kedah, Sintok, Malaysia \\ ${ }^{3}$ Department of Mathematics, College of arts and Sciences Tabarjal, Jouf Univeristy, Sakakah, Saudi Arabia \\ ${ }^{4}$ Department of Mathematics, Faculty of Science, Yarmouk University, Irbid, Jordan
}

\begin{tabular}{l} 
Article Info \\
\hline Article history: \\
Received Apr 14, 2021 \\
Revised Aug 16, 2021 \\
Accepted Sep 10, 2021
\end{tabular}

\section{Keywords:}

Laplace transform MRPSM

Pade approximant RPSM

System of multi-pantograph

Type differential equation

\section{Corresponding Author:}

Nidal Anakira

Department of Mathematics, Faculty of Science and Technology, Irbid National University

2600 Irbid, Jordan

Email: alanaghreh_nedal@yahoo.com

\begin{abstract}
In this paper, a modified procedure based on the residual power series method (RPSM) was implemented to achieve approximate solution with high degree of accuracy for a system of multi-pantograph type delay differential equations (DDEs). This modified procedure is considered as a hybrid technique used to improve the curacy of the standard RPSM by combining the RPSM, Laplace transform and Pade approximant to be a powerful technique that can be solve the problems directly without large computational work, also even enlarge domain and leads to very accurate solutions or gives the exact solutions which is consider the best advantage of this technique. Some numerical applications are illustrated and numerical results are provided to prove the validity and the ability of this technique for this type of important differential equation that appears in different applications in engineering and control system.
\end{abstract}

This is an open access article under the CC BY-SA license.

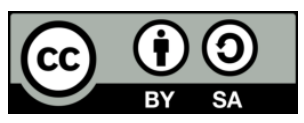

\section{INTRODUCTION}

In this study, the following system of multi-pantograph type DDEs will be considered.

$$
\begin{aligned}
& u_{1}^{\prime}(t)=\beta_{1} u_{1}(t)+f_{1}\left(t, u_{1}\left(\alpha_{11} t\right), u_{2}\left(\alpha_{11} t\right), \ldots, u_{n}\left(\alpha_{11} t\right)\right) \\
& u^{\prime}{ }_{2}(t)=\beta_{2} u_{2}(t)+f_{2}\left(t, u_{1}\left(\alpha_{21} t\right), u_{2}\left(\alpha_{21} t\right), \ldots, u_{n}\left(\alpha_{21} t\right)\right)
\end{aligned}
$$

Subject to the following initial conditions

$$
u_{i}\left(t_{0}\right)=u_{i}, \quad t_{0}<t \leq T
$$

where, $\beta_{i}, u_{i}$ are finite constant, $f_{i}$, are analytical functions such that

$$
0<\alpha_{i j} \leq 1, \quad \alpha_{i j}=1,2, \ldots, n .
$$

and $u_{i}(t), i=1,2, \ldots, n$. are unknown functions that will be determined. In recent years, numerous numerical and approximate analytical studies related to the delay differential equations (DDEs) have been appeared especially the multi-pantograph type DDEs which is an important types of DDEs that appears in 
various applications of engineering and branches of applied science such as electronic systems, population dynamics, dynamical systems, quantum mechanics. Obtaining analytical solutions of these kinds of DDEs are generally difficult. Therefore, in literature, many powerful and effective numerical procedures are used to approximate the solutions of multi-pantograph DDEs, for examples, optimal homotopy asymptotic method (OHAM) and its modifications are used to obtain approximate solutions for multi-pantograph time DDEs [1]-[7]. A domain decomposition method (ADM), differential transformation method (DTM) are also another approaches which they employed to solve multi-pantograph equations [8]. A collocation method is also one of the methods which is employed to find an approximate solution of a system of multi pantograph type delay differential [9]. Moreover, a number of solution methods have been proposed and employed for the approximate solutions of multi-pantograph type DDEs [10]-[13]. Mathematical modeling of physicalworld phenomena leads to linear or nonlinear ordinary or partial differential equations in different branches of applied science and engineering. It is well-known that the solution of the nonlinear one is not easy either numerically or theoretically and even more difficult to establish a real model for the nonlinear problem. Therefore, a researcher has put their efforts and attention to develop and construct analytical and numerical methods to study and analyze the solution of the differential equations trying to provide highly accurate solution which is closed form to the analytic solution or give it.

The residual power series method (RPSM) is one of an effective and easy power series procedure which was used in a large scale in the last few years for various forms of strongly linear and nonlinear differential equations and fractional differential equations without any limitations or restrictions such as linearization, discretization and perturbation [14]-[20]. Also, it has been successfully employed to handle the numerical solution of a highly nonlinear singular Lane-Emden equation type [21]. The fundamental motivation of this paper is based on improving the accuracy of the RPSM using an alternative procedure for the first time that modify the series solution of the RPSM throughout using the Laplace transformation to the truncated RPSM solution then convert the transformed series into a meromorphic function by using Pade approximants, lastly, we apply the inverse of the Laplace transformation to get the required solution of the given problem. This method is easy, and does not requires big efforts to achieve accurate results with high performance.

The rest of this paper is arranged as follows. Section 2 is conducted to illustrate the fundamental concept of the RPSM along with the method analysis besides to preliminary of the Pade approximants. In section 3 numerical examples are given to prove the capacity of the discussed procedure by comparing the results which indicate that only a few terms are required to deduce the exact solutions. Finally, conclusions of this work are formulated in the last section.

\section{RESEARCH METHOD}

\subsection{Residual power series method (RPSM)}

The aim of this part is to give an overview and some properties of the RPSM procedure, which will be employed in order to get series solution of a system of multi-pantograph type DDEs [22]-[24]. The RPSM provide us the solutions of the given system in a form of power series expansion about the initial point $t=t_{0}$ to receive our objective, we assume the solutions in the form of (3):

$$
u_{i}(t)=\sum_{m=0}^{\infty} u_{i, m}(t), i=1,2, \ldots n,
$$

where $u_{i, m}(t)$ are the order of approximations such that $u_{i, m}(t)=c_{i, m}\left(t-t_{0}\right)^{m}$. Then, by substituting the initial guesses $u_{i, 0}\left(t_{0}\right)=u_{i}^{(m)}\left(t_{0}\right)=c_{i, 0}$, which are the initial conditions (2), using $m=0$, into $u_{i}(t), i=1,2$, yield to the approximate solution for the system of multi-pantograph equations $u_{i}(t)=u_{i, 0}\left(t_{0}\right)+\sum_{m=0}^{\infty} u_{i, m}(t), i=1,2, \ldots n$, whereas $u_{i, m}(t)$ for $m=1,2, \ldots, k$, can be obtained from the following $\mathrm{k}^{\text {th }}$-truncated series.

$$
u_{i, k}(t)=\sum_{m=0}^{k} c_{i, m}\left(t-t_{0}\right)^{m}, i=1,2, \ldots n,
$$

To use the RPSM, we write the system of (1) and (2) in the form given:

$$
\begin{aligned}
& u^{\prime}{ }_{1}(t)-\beta_{1} u_{i}(t)-f_{1}\left(t, u_{1}\left(\alpha_{i 1} t\right), u_{2}\left(\alpha_{i 2} t\right), \ldots, u_{n}\left(\alpha_{i n} t\right)\right) \\
& u^{\prime}{ }_{2}(t)-\beta_{2} u_{i}(t)-f_{2}\left(t, u_{1}\left(\alpha_{i 1} t\right), u_{2}\left(\alpha_{i 2} t\right), \ldots, u_{n}\left(\alpha_{i n} t\right)\right)
\end{aligned}
$$

The $\mathrm{k}^{\text {th }}$ and the $\infty^{\text {th }}$ residual functions are given respectively, in the following manner 


$$
\operatorname{Res}_{i}^{k}(t)=u_{i, k}^{\prime}(t)-\beta_{i} u_{i, k}(t)-f_{i}\left(t, u_{1, k}\left(\alpha_{i 1} t\right), u_{2, k}\left(\alpha_{i 2} t\right), \ldots, u_{n, k}\left(\alpha_{i n} t\right), i=1,2, \ldots, n\right.
$$

and

$$
\begin{aligned}
& \operatorname{Res}_{i}^{\infty}(t)=\lim _{k \rightarrow \infty} \operatorname{Res}_{i}^{k}(t)=u_{i}^{\prime}(t)-\beta_{i} u_{i}(t)- \\
& f_{i}\left(t, u_{1}\left(\alpha_{i 1} t\right), u_{2}\left(\alpha_{i 2} t\right), \ldots, u_{n}\left(\alpha_{i n} t\right), i=1,2, \ldots, n\right.
\end{aligned}
$$

Clearly, we see that $\operatorname{Res}_{i}^{\infty}(t)=0$ for each $t \in\left(t_{0}, T\right)$, are infinitely differentiable functions at $t=t_{0}$. Moreover, $\frac{d^{m}}{d t^{m}} \operatorname{Res}_{i}^{\infty}\left(t_{0}\right)=\frac{d^{m}}{d t^{m}} \operatorname{Res}_{i}^{k}\left(t_{0}\right)=0, m=1,2, \ldots, k$, which is a fundamental rule in the RPSM and its applications. In particular, $\frac{d^{k-1}}{d t^{k-1}} \operatorname{Res}_{i}^{\infty}\left(t_{0}\right)=\frac{d^{k-1}}{d t^{k-1}} \mathcal{R e s}_{i}^{k}\left(t_{0}\right)=0, i=1,2, \ldots, n, k=1,2, \ldots$.

Then by substituting the $\mathrm{k}^{\text {th }}$-truncated series $u_{i, k}(t)$ into (6) we get

$$
\begin{aligned}
& \operatorname{Res}_{i}^{k}(t)=\sum_{m=1}^{k} m c_{i, m}\left(t-t_{0}\right)^{m-1} \\
& -\beta_{i} \sum_{m=0}^{k} c_{i, m}\left(t-t_{0}\right)^{m}-f_{i}\left(t, \sum_{m=0}^{k} c_{1} m\left(\alpha_{i 1} t-t_{0}\right)^{m},\right. \\
& \sum_{m=0}^{k} c_{2} m\left(\alpha_{i 2} t-t_{0}\right)^{m}, \ldots, \sum_{m=0}^{k} c_{n, m}\left(\alpha_{i n} t-t_{0}\right)^{m}, i=1,2, \ldots, n
\end{aligned}
$$

Hence, depend on $\operatorname{Res}_{i}^{1}\left(t_{0}\right)=0, i=1,2, \ldots, n$. Using $t=t_{0}=0$ and $k=1$ into (8) yields to

$$
c_{i, 1}=\beta_{i} c_{i, 0}+f_{i}\left(t_{0}, c_{1,0}, c_{2,0}, \ldots, c_{n, 0}\right)=\beta_{i} u_{i, 0}+f_{i}\left(0, \bar{u}_{i, 0}\right), i=1,2, \ldots, n
$$

where $f_{i}\left(0, \bar{u}_{i, 0}\right)=f_{i}\left(0, u_{1,0}, u_{2,0}, \ldots, u_{n, 0}\right)$. Using (4), the first approximate solutions of the system (1) and (2) will be written in the following form (10).

$$
u_{i, 1}(t)=u_{i, 0}+\left(\beta_{i} u_{i, 0}+f_{i}\left(0, \bar{u}_{i, 0}\right)\right)\left(t-t_{0}\right), i=1,2, \ldots, n
$$

The second-order approximate solutions can be obtained by using $k=2$ and $t_{0}=0$ such that $u_{i, 2}(t)=\sum_{m=0}^{2} c_{i, m} t^{m}$. Then, by differentiate with respect to $t$, we have (11).

$$
\begin{aligned}
& \frac{d}{d t} \operatorname{Res}_{i}^{2}(0)=2 c_{i, 2}-\beta_{i} c_{i, 1}-\frac{d}{d t}\left(f _ { i } \left(t, \sum_{m=0}^{2} c_{1, m} \alpha_{i 1}^{m} t^{m},\right.\right. \\
& \left.\left.\sum_{m=0}^{2} c_{2, m} \alpha_{i 2}^{m} t^{m}, \ldots, \sum_{m=0}^{2} c_{n, m} \alpha_{i n}^{m} t^{m}\right)\right), i=1,2, \ldots, n
\end{aligned}
$$

Based on the fact that the $\frac{d^{k-1}}{d t^{k-1}} \operatorname{Res}_{i}^{k}(0)=0, i=1,2, \ldots, n$, the values of $c_{i, 2}$ are given by (12),

$$
\begin{aligned}
& c_{i, 2}=\frac{1}{2}\left(\beta_{i} c_{i, 1}+\frac{d}{d t}\left[f _ { i } \left(t, \sum_{m=0}^{2} c_{1, m} \alpha_{i 1}^{m} t^{m},\right.\right.\right. \\
& \left.\left.\sum_{m=0}^{2} c_{2, m} \alpha_{i 2}^{m} t^{m}, \ldots, \sum_{m=0}^{2} c_{n, m} \alpha_{i n}^{m} t^{m}\right]_{t=0}\right), i=1,2, \ldots, n
\end{aligned}
$$

Therefore, the second approximate solutions will be (13),

$$
\begin{aligned}
& u_{i, 2}=u_{i, 0}+\left(\beta_{i} u_{i, 0}+f_{i}\left(0, \bar{u}_{i, 0}\right)\right)\left(t-t_{0}\right)+ \\
& \frac{1}{2}\left(\beta_{i} c_{i, 1}+g_{i}\left(0, \bar{u}_{i, 1}\right)\left(t-t_{0}\right)^{2}, i=1,2, \ldots, n\right.
\end{aligned}
$$

where $g_{i}\left(0, \bar{u}_{i, 1}\right)=\frac{d}{d t}\left[f_{i}\left(t, \sum_{m=0}^{2} c_{1, m} \alpha_{i 1}^{m} t^{m}, \sum_{m=0}^{2} c_{2, m} \alpha_{i 2}^{m} t^{m}, \ldots, \sum_{m=0}^{2} c_{n, m} \alpha_{i n}^{m} t^{m}\right]_{t=0}\right), i=1,2, \ldots, n$ and $c_{i, 1}$, are given in (9) for $i=1,2, \ldots, n$. We follow the same procedure to generate a sequence of approximate solutions $u_{i, k}(t)$ for the system (1) and (2) Furthermore, high accuracy can be achieved by increasing the order of the approximations. For this regards, we let $\operatorname{Rem}_{i}^{k}(t)$ to denote the difference between $u_{i}(t)$ and its $k t h$ Taylor polynomial, that is, here the functions $\mathcal{R e m}_{i}^{k}(t)$ represent the $k t h$ remainder of the Taylor series of $u_{i}(t)$.

$$
\mathcal{R e m}_{i}^{k}(t)=u_{i}(t)+u_{i, k}(t)=\sum_{m=k+1}^{\infty} \frac{1}{m !} u_{i}^{(m)}\left(t_{0}\right)\left(t-t_{0}\right)^{m}, i=1,2, \ldots, n
$$




\subsection{Padè approximation}

The $[L / M]$ Padè approximants [25]-[27] of a function $u(x)$ is defined by:

$$
\left[\frac{L}{M}\right]=\frac{P_{L}(t)}{Q_{M}(t)}
$$

where $P_{L}(t)$ and $Q_{M}(t)$ are polynomials of degrees at most $L$ and $M$, respectively. The general form of the power series is

$$
u(t)=\sum_{i=1}^{\infty} a_{i} t^{i}
$$

The coefficients of the $P_{L}(t)$ and $Q_{M}(t)$ polynomials are obtained from:

$$
u(t)-\frac{P_{L}(t)}{Q_{M}(t)}=O\left(t^{L+M+1}\right)
$$

when the fraction of the numerator and denominator $\frac{P_{L}(t)}{Q_{M}(t)}$ is multiplying by a nonzero constant the fractional values remain unchanged, then we can define the normalization condition as (16):

$$
Q_{M}(0)=1
$$

It can be noted that $\mathrm{P}_{\mathrm{L}}(\mathrm{t})$ and $\mathrm{Q}_{\mathrm{M}}(\mathrm{t})$ have no public factors. If we repsents the coefficient of $\mathrm{P}_{\mathrm{L}}(\mathrm{t})$ and $\mathrm{Q}_{\mathrm{M}}(\mathrm{t})$ as (17):

$$
\left\{\begin{array}{c}
P_{L}(t)=p_{0}+p_{1} t+p_{2} t^{2}+\cdots+p_{L} t^{L} \\
Q_{M}(t)=q_{0}+q_{1} t+q_{2} t^{2}+\cdots+q_{M} t^{M}
\end{array}\right.
$$

Then, by (16) and (17), we can multiply (15) by $\mathrm{Q}_{\mathrm{M}}(\mathrm{t})$, to linearizes the coefficient equations. We can write out (18) in more detail as (18):

$$
\left\{\begin{array}{c}
a_{L+1}+a_{L} q_{1}+\cdots+a_{L-M+1} q_{M}=0 \\
a_{L+2}+a_{L+1} q_{1}+\cdots+a_{L-M+2} q_{M}=0 \\
\cdot \\
a_{L+M}+a_{L+M-1} q_{1}+\cdots+a_{L} q_{M}=0
\end{array}\right.
$$

To obtain the solutions of these equations, we begin with (18), which is consider as a set of linear equations for all of the unknown $\mathrm{q}^{\prime} \mathrm{s}$. Once the $\mathrm{q}^{\prime} \mathrm{s}$ are known, then (19) gives an explicit formula for the unknown $\mathrm{p}^{\prime}$ s, which complete the solution. If (18) and (19) are non-singular, then we may solve them direct and we obtain (21) [23], where (21) holds, and if the lower index on a sum exceeds the upper, the sum is replaced by zero:

$$
\left\{\begin{array}{c}
\mathrm{a}_{0}=\mathrm{p}_{0} \\
\mathrm{a}_{0}+\mathrm{a}_{0} \mathrm{q}_{1}=\mathrm{p}_{1} \\
\mathrm{a}_{2}+\mathrm{a}_{1} \mathrm{q}_{1}+\mathrm{a}_{0} \mathrm{q}_{2}=\mathrm{p}_{2} \\
\cdot \\
\cdot \\
\mathrm{a}_{\mathrm{L}}+\mathrm{a}_{\mathrm{L}-1} \mathrm{q}_{1}+\cdots+\mathrm{a}_{0} \mathrm{q}_{\mathrm{L}}=\mathrm{p}_{\mathrm{L}}
\end{array}\right.
$$

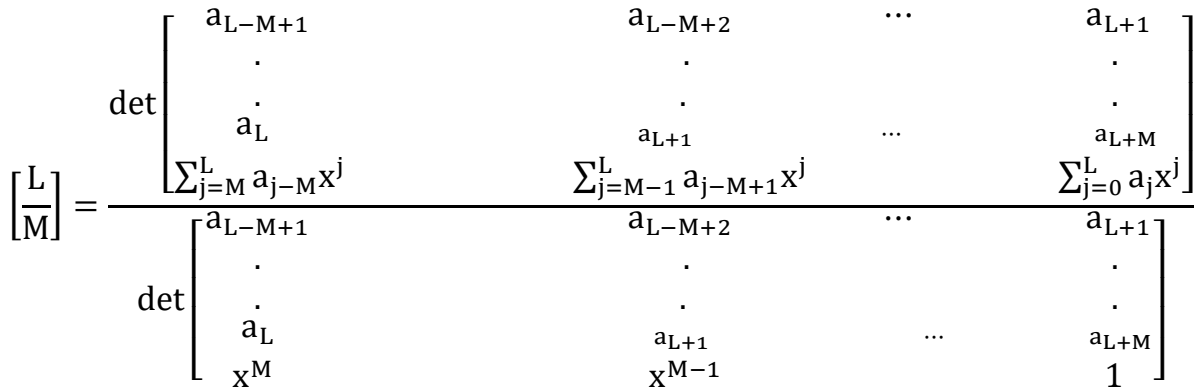


Therefore, the Pade approximants diagonal matrix of different order can be obtained using Mathematica software or like MATLAB and son.

\section{RESULTS AND DISCUSSION}

\subsection{Example 1}

The following system of multi-pantograph type delay differential equations considered [21].

$$
\begin{aligned}
& \mathrm{u}_{1}^{\prime}(\mathrm{t})-\mathrm{u}_{1}(\mathrm{t})+\mathrm{u}_{2}(\mathrm{t})-\mathrm{u}_{1}\left(\frac{\mathrm{t}}{2}\right)=\mathrm{g}_{1}(\mathrm{t}) \\
& \mathrm{u}_{2}(\mathrm{t})+\mathrm{u}_{1}(\mathrm{t})+\mathrm{u}_{2}(\mathrm{t})+\mathrm{u}_{2}\left(\frac{\mathrm{t}}{2}\right)=\mathrm{g}_{2}(\mathrm{t}) \\
& \mathrm{u}_{1}(0)=1 \quad \mathrm{u}_{2}(0)=1
\end{aligned}
$$

where

$$
g_{1}(t)=e^{-t}-e^{-\frac{t}{2}} \quad g_{2}(t)=e^{t}+e^{-\frac{t}{2}}
$$

To solve this system by using RPSM, we start with the initial conditions $\mathrm{u}_{1,0}=1$ and $\mathrm{u}_{2,0}=1$, as an initial guesses, then, we consider the kth-truncated series solutions of $u_{1, k}(t)$ and $u_{2, k}(t)$ in form (24):

$$
\begin{aligned}
& u_{1, k}(t)=\sum_{m=0}^{k} c_{1, m} t^{m}=1+c_{1,1} t+c_{1,2} t^{2}+c_{1,3} t^{3}+\cdots, c_{1, k} t^{k} \\
& u_{2, k}(t)=\sum_{m=0}^{k} c_{2, m} t^{m}=1+c_{2,1} t+c_{2,2} t^{2}+c_{2,3} t^{3}+\cdots, c_{2, k} t^{k}
\end{aligned}
$$

Now, the unknown coefficients $c_{i, m}, i=1,2 . m=1,2, \ldots, k$ can be obtained by formulated the following $\mathrm{k}^{\text {th }}$ residual functions

$$
\begin{aligned}
& \operatorname{Res}_{1}^{k}(t)=\sum_{m=1}^{k} m c_{1, m} t^{m-1}-\sum_{m=0}^{k} c_{1, m} t^{m}+\sum_{m=0}^{k} c_{2, m} t^{m} \\
& -\sum_{m=0}^{k} c_{1, m}\left(\frac{t}{2}\right)^{m}-e^{-t}+e^{\frac{t}{2}} \\
& \operatorname{Res}_{2}^{k}(t)=\sum_{m=1}^{k} m c_{2, m} t^{m-1}+\sum_{m=0}^{k} c_{1, m} t^{m}+\sum_{m=0}^{k} c_{2, m} t^{m} \\
& +\sum_{m=0}^{k} c_{2, m}\left(\frac{t}{2}\right)^{m}-e^{t}+e^{-\frac{t}{2}},
\end{aligned}
$$

The first-order approximation solution $u_{1,1}(t)=1-t$ and $u_{2,1}(t)=t$ can be obtained based on the above residual function by using $\mathrm{k}=1$ to get

$$
\begin{aligned}
& \operatorname{Res}_{1}^{1}(t)=c_{1,1}+\left(c_{2,1}-\frac{3}{2} c_{1,1}\right) t-e^{-t}+e^{\frac{t}{2}}-1 \\
& \operatorname{Res}_{2}^{1}(t)=c_{2,1}+\left(c_{1,1}+\frac{3}{2} c_{1,1}\right) t-e^{-\frac{t}{2}}-e^{-t}+3
\end{aligned}
$$

Based on the fact that $\frac{d}{d t} \operatorname{Res}_{1}^{1}(0)=\frac{d}{d t} \operatorname{Res}_{2}^{1}(0)=0$, we have $c_{1,1}=1$ and $c_{2,2}=-1$, which yields to the first order approximate solution $u_{1,1}=1+t$ and $u_{2,1}=1-t$. The values of the coefficients $c_{1,2}$ and $c_{2,2}$ can be found by differentiate both sides of (25) with respect to $\mathrm{t}$ when $\mathrm{k}=2$ and then substituting $\mathrm{t}=0$ to be $c_{1,2}=$ $\frac{1}{2}$ and $c_{2,2}=-\frac{1}{2}$ to obtain the following second order approximate solution $u_{1,2}=1+t+\frac{1}{2} t$, and $u_{2,2}=1+$ $t-\frac{1}{2} t$. By follow the same proceeder, the $5^{\text {th }}$ order approximate solutions for the given system become

$$
\begin{aligned}
& u_{1, k}(t)=1+t+\frac{t^{2}}{2 !}+\frac{t^{3}}{3 !}+\frac{t^{4}}{4 !}+\frac{t^{5}}{5 !}+\frac{t^{6}}{6 !}+\cdots \\
& u_{2, k}(t)=1-t+\frac{t^{2}}{2 !}-\frac{t^{3}}{3 !}+\frac{t^{4}}{4 !}-\frac{t^{5}}{5 !}+\frac{t^{6}}{6 !}-\cdots
\end{aligned}
$$

which leads to the analytic form of the solution in the limit of infinity terms of the order of the approximation. To improve the efficiency of the solutions procedure and get high accuracy we will use an effective and powerful modification based on RPSM truncated series solutions by employing the Laplace transform to the first four terms of (27) to obtain. 


$$
L\left\{u_{1,5}(t)\right\}=\frac{1}{s}+\frac{1}{s^{2}}+\frac{1}{s^{3}}+\frac{1}{s^{4}}, L\left\{u_{2,5}(t)\right\}=\frac{1}{s}-\frac{1}{s^{2}}+\frac{1}{s^{3}}-\frac{1}{s^{4}}
$$

Consider $s=\frac{1}{z}$, yields

$$
L\left\{u_{1,5}(t)\right\}=z+z^{2}+z^{3}+z^{4}, L\left\{u_{1,5}(t)\right\}=z-z^{2}+z^{3}-z^{4}
$$

Then, using the Pad approximants of $\left[\frac{4}{4}\right]$.

$$
\begin{aligned}
& L u=\frac{z}{1-z} \\
& L u=\frac{z}{1+z}
\end{aligned}
$$

Noting that $z=\frac{1}{s}$, and by using the inverse of the Laplace transform, we have

$$
\begin{aligned}
& u_{1}(t)=e^{t} \\
& u_{2}(t)=e^{-t}
\end{aligned}
$$

which is the exact form of the above system.

\subsection{Example 2}

Consider the following nonlinear system of multi-pantograph type delay equation [21]

$$
\begin{aligned}
& u_{1}^{\prime}(t)=u_{1}(t)-e^{-t} \cos \left(\frac{t}{2}\right) u_{2}\left(\frac{t}{2}\right)-2 e^{\left(-\frac{3}{4} t\right)} \cos \left(\frac{t}{2}\right) \sin \left(\frac{t}{2}\right), \\
& u_{2}^{\prime}(t)=e^{t} u_{1}^{2}\left(\frac{t}{2}\right)-u_{2}^{2}\left(\frac{t}{2}\right)
\end{aligned}
$$

Subject to given initial conditions

$$
u_{1}(0)=1, \quad u_{2}(0)=0
$$

To obtain the approximate solution using RPSM, we will start with the initial conditions

$$
u_{1,0}(t)=1, \quad u_{2,0}(t)=0
$$

The kth-truncated series formula (3) for this example by using (17) is

$$
\begin{aligned}
& u_{1, k}(t)=\sum_{m=0}^{k} c_{1, m} t^{m}=1+c_{1,1} t+c_{1,2} t^{2}+c_{1,3} t^{3}+\cdots, c_{1, k} t^{k}, \\
& u_{2, k}(t)=\sum_{m=0}^{k} c_{2, m} t^{m}=1+c_{2,1} t+c_{2,2} t^{2}+c_{2,3} t^{3}+\cdots, c_{2, k} t^{k}
\end{aligned}
$$

whereas the $\mathrm{k}^{\text {th }}$ residual function $\operatorname{Res}_{i}^{k}(t)$, where $i=1,2$, is

$$
\begin{aligned}
& \operatorname{Res}_{1}^{k}(t)=\sum_{m=1}^{k} m c_{1, m} t^{m-1}+\sum_{m=0}^{k} c_{1, m} t^{m}+e^{-t} \cos \left(\frac{t}{2}\right) \sum_{m=1}^{k} c_{2, m}\left(\frac{t}{2}\right)^{m} \\
& +2 e^{\left(-\frac{3}{4} t\right)} \cos \left(\frac{t}{2}\right) \sin \left(\frac{t}{4}\right) \sum_{m=0}^{k} c_{1, m}\left(\frac{t}{4}\right)^{m}, \\
& \operatorname{Res}_{2}^{k}(t)=\sum_{m=1}^{k} m c_{2, m} t^{m-1}+e^{t}\left(\sum_{m=0}^{k} c_{1, m}\left(\frac{t}{2}\right)^{m}\right)^{2}+\left(\sum_{m=0}^{k} c_{2, m}\left(\frac{t}{2}\right)^{m}\right)^{2}
\end{aligned}
$$

when $k=1$, we get the first order RPS approximate solution $u_{1,1}(t)=1-t$ and $u_{2,1}(t)=t$. The 2nd orderRPS approximate solution can be obtained by differentiate (36) and using $t=0, c_{1,0}=1$ and $c_{2,0}=0$. We follow the same procedure up to the $\mathrm{k}^{\text {th }}$ order based on the $\frac{d^{k-1}}{d x^{k-1}}\left(\operatorname{Res}_{i}^{k}(0)=0\right)$; $i=1,2,10$ th order approximate solution is

$$
\begin{aligned}
& u_{1,10}(t)=1-t+\frac{t^{3}}{3}-\frac{t^{4}}{6}+\frac{t^{5}}{30}-\frac{t^{7}}{630}+\frac{t^{8}}{2520} \\
& u_{1,10}(t)=t+\frac{t^{3}}{6}-\frac{t^{5}}{120}+\frac{t^{7}}{5040}-\frac{t^{9}}{362880}
\end{aligned}
$$


which leads to the analytic form of the solution in the limit of infinity terms of the order of the approximation. To improve the efficiency of the solutions procedure and get high accuracy we will use an effective and powerful modification based on RPSM truncated series solutions by employing the Laplace transform to the first four terms of (37) to obtain.

$$
\begin{aligned}
& L\left\{u_{1,5}(t)\right\}=\frac{1}{s}-\frac{1}{s^{2}}+\frac{2}{s^{4}}-\frac{4}{s^{5}}+\frac{4}{s^{6}} \\
& L\left\{u_{2,5}(t)\right\}=\frac{1}{s^{2}}-\frac{1}{s^{4}}+\frac{1}{s^{6}}-\frac{1}{s^{8}}+\frac{1}{s^{10}}
\end{aligned}
$$

For the simplicity purpose, let $s=\frac{1}{z}$, yields to

$$
\begin{aligned}
& L\left\{u_{1,5}(t)\right\}=z-z^{2}+2 z^{4}-4 z^{5}+4 z^{6} \\
& L\left\{u_{1,5}(t)\right\}=z^{2}+z^{4}-z^{6}+z^{8}+z^{10}
\end{aligned}
$$

Now, we use the Pad approximants of $\left[\frac{5}{5}\right]$.

$$
L u=\frac{z^{2}+z}{2 z^{2}+2 z+1}, L u=\frac{z^{2}}{z^{2}+1}
$$

Consider $z=\frac{1}{s}$, and by using the inverse of the Laplace transform to obtain

$$
\begin{aligned}
& u_{1}(t)=e^{-t} \cos (t) \\
& u_{2}(t)=\sin t
\end{aligned}
$$

which is the exact form of the above system.

\subsection{Example 3}

Consider the nonlinear system of multi-pantograph type delay equation [21].

$$
\begin{aligned}
& u_{1}^{\prime}(t)=2 u_{2}\left(\frac{t}{2}\right)+u_{3}(t) t \cos \left(\frac{t}{2}\right) \\
& u_{2}^{\prime}(t)=1-t \sin t-2 u_{3}^{2}\left(\frac{t}{2}\right) \\
& u_{3}^{\prime}(t)=u_{2}(t)-u_{1}(t)-t \cos t
\end{aligned}
$$

Subject to the given initial conditions.

$$
u_{1}(0)=-1 \quad u_{2}(0)=0 \quad u_{3}(0)=0
$$

To obtain the approximate solution using RPSM, we start with the initial approximation $u_{1,0}(0)=-1 \quad u_{2,0}(0)=0 \quad u_{3,0}(0)=0$. Then we follow the same procedure which applied in the previous examples, to obtain the following truncated RPS series approximation

$$
\begin{aligned}
& u_{1, k}(t)=\sum_{m=0}^{k} c_{1, m} t^{m}=-1+\frac{t^{2}}{2}-\frac{t^{4}}{24}+\frac{t^{6}}{720}-\frac{t^{8}}{40320}+\frac{t^{10}}{3628800}+\cdots, \\
& u_{2, k}(t)=\sum_{m=0}^{k} c_{2, m} t^{m}=t-\frac{t^{3}}{2}+\frac{t^{5}}{24}-\frac{t^{7}}{720}-\frac{t^{9}}{40320}+\cdots \\
& u_{3, k}(t)=\sum_{m=0}^{k} c_{3, m} t^{m}=t-\frac{t^{3}}{6}+\frac{t^{5}}{120}-\frac{t^{7}}{5040}+\frac{9}{362880}+\cdots
\end{aligned}
$$

which leads to the analytic form of the solution in the limit of infinity terms of the order of the approximation. To improve the efficiency of the solutions procedure and get high accuracy we will use an effective and powerful modification based on RPSM truncated series solutions by employing the Laplace transform to the first four terms of (44) to obtain:

$$
\begin{aligned}
& L\left\{u_{1,4}(t)\right\}=-\frac{1}{s^{9}}+\frac{1}{s^{7}}-\frac{1}{s^{5}}+\frac{1}{s^{3}}-\frac{1}{s^{\prime}} \\
& L\left\{u_{2,4}(t)\right\}=\frac{9}{s^{10}}-\frac{7}{s^{8}}+\frac{5}{s^{6}}-\frac{3}{s^{4}}+\frac{1}{s^{2}} \\
& L\left\{u_{2,4}(t)\right\}=\frac{1}{s^{10}}-\frac{1}{s^{8}}+\frac{1}{s^{6}}-\frac{1}{s^{4}}+\frac{1}{s^{2}}
\end{aligned}
$$


For the purpose of simplicity, let $=\frac{1}{z}$, yields.

$$
\begin{aligned}
& L\left\{u_{1,5}(t)\right\}=-z^{9}+z^{7}-z^{5}+z^{3}-z, \\
& L\left\{u_{2,5}(t)\right\}=9 z^{10}-7 z^{8}+5 z^{6}-3 z^{4}+z^{2}, \\
& L\left\{u_{3,5}(t)\right\}=z^{10}-z^{8}+z^{6}-z^{4}+z^{2}
\end{aligned}
$$

Now, we use the Pad approximants of $\left[\frac{5}{5}\right]$.

$$
\begin{aligned}
& L_{u}=-\frac{z}{z^{2}+1} \\
& L_{u}=\frac{z^{2}-z^{4}}{z^{4}-2 z^{2}+1}, \\
& L_{u}=\frac{z^{2}}{z^{2}+1}
\end{aligned}
$$

In (47) recalling that $z=\frac{1}{s}$, and then applying the inverse Laplace transform to obtain

$$
\begin{aligned}
& u_{1}(t)=-\cos (t) \\
& u_{2}(t)=t \cos (t) \\
& u_{3}(t)=\sin (t)
\end{aligned}
$$

which is the exact form of the above system. From the numerical results obtained, we saw that the proposed procedure provides us high accurate solutions closed to the exact one. This can be obtained by using a few numbers of iterations of the standard RPSM solutions, this advantage overcomes the difficulties that its need to compute more iterations to get more accurate solutions to improve efficiency of the standard RPSM that is noted from the absolute errors represented by Figure 1 to Figure 3.

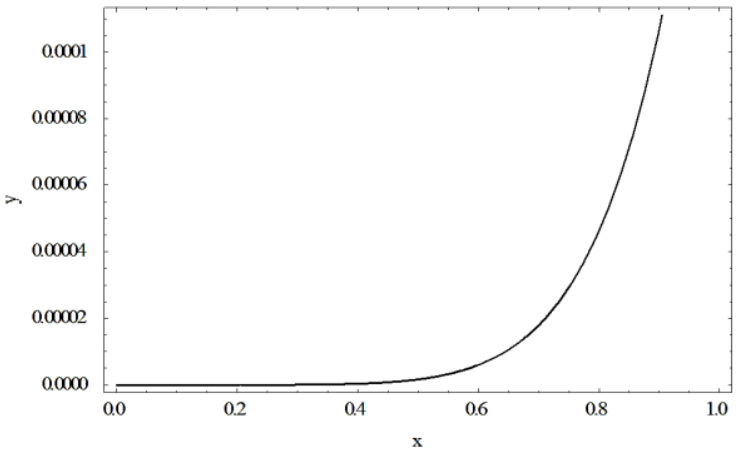

(a)

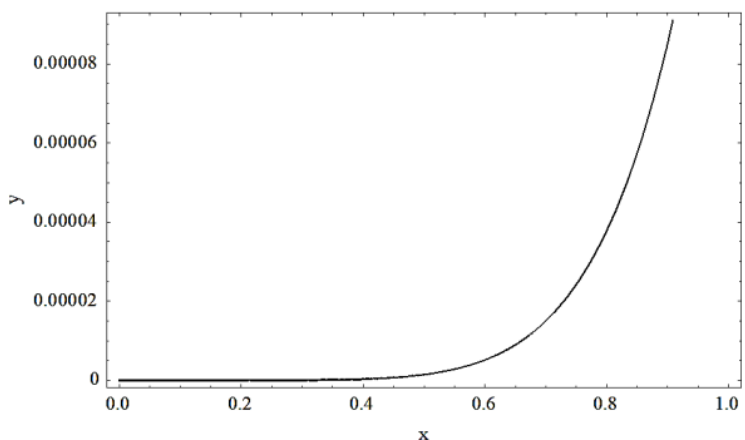

(b)

Figure 1. Absolute errors related to $(a) u_{1}(t)$ and $(b) u_{2}(t)$ of example 1

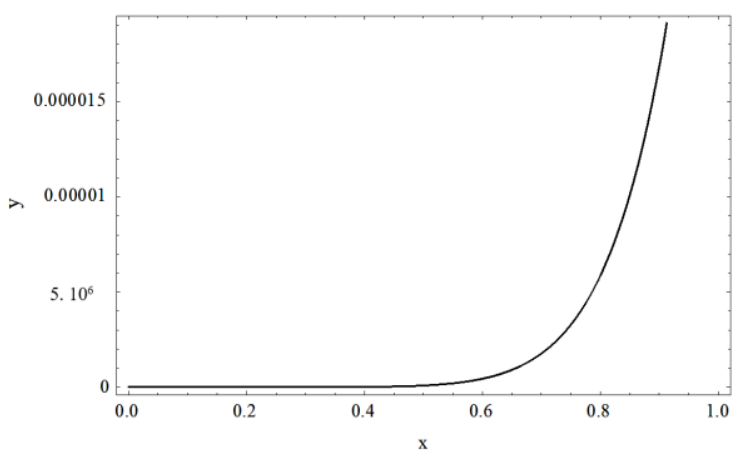

(a)

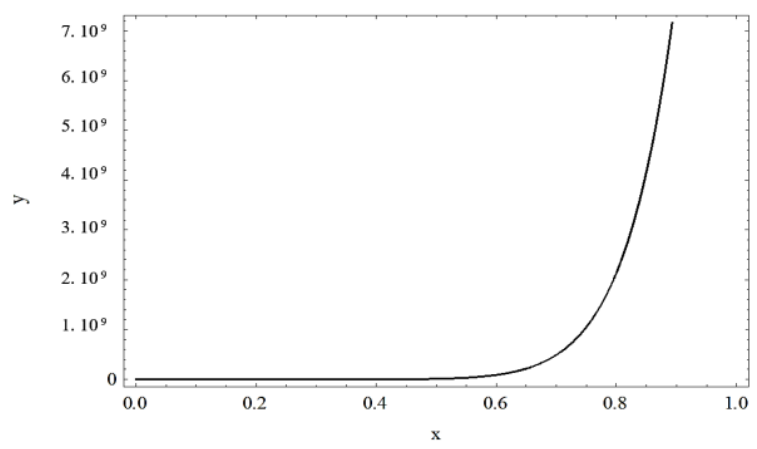

(b)

Figure 2. Absolute errors related to (a) $u_{1}(t)$ and (b) $u_{2}(t)$ of example 2 


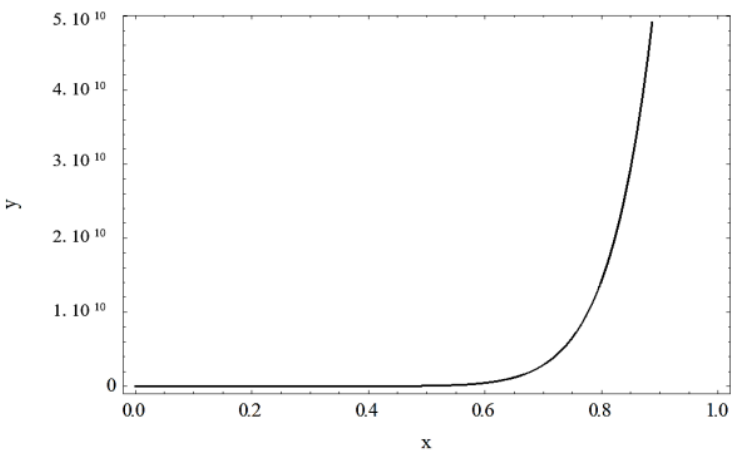

(a)

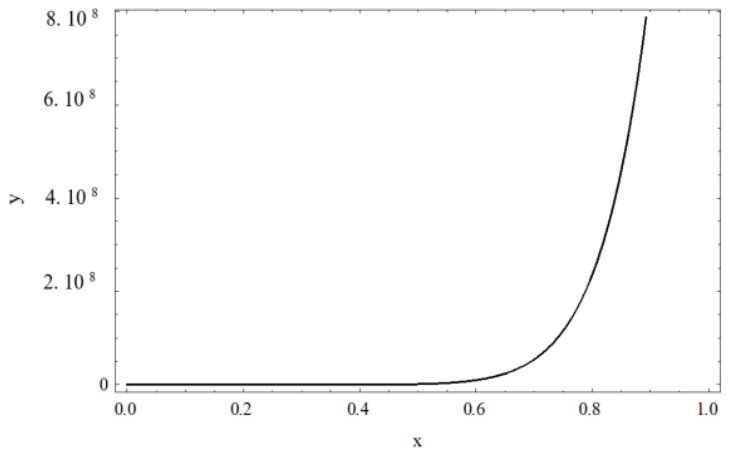

(b)

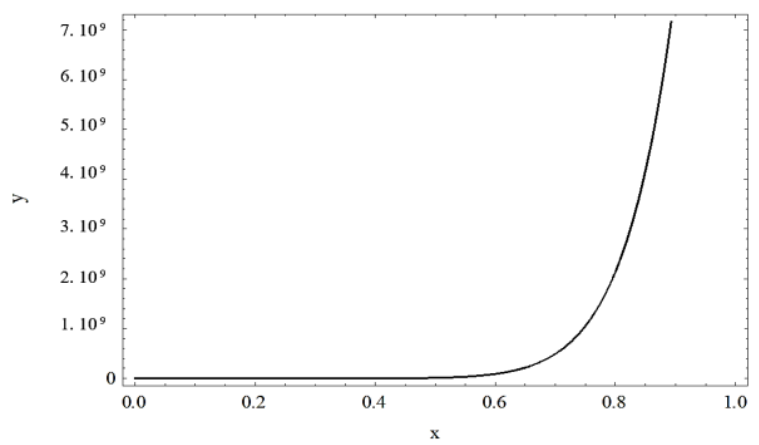

(c)

Figure 3. Absolute errors related to (a) $u_{1}(t)$ and (b) $u_{2}(t)$ of example 1 , and (c) $u_{3}(t)$ of example 3

\section{CONCLUSION}

In this research study, a new procedure based on the RPSM was proposed to solve system of multi-pantograph type DDEs. This procedure is effective, reliable and has a distinct advantage over the other methods represented by it is valid for the strongly nonlinear problems. It has been shown throughout the illustration examples and the comparison with of the numerical results reported in the literature that the MRPSM procedure has the sufficient to obtain the exact analytical solution using only few terms of the RPSM truncated series solution. This leads to conclude that this procedure is a powerful approach and a promising tool for solving this kind of differential equations.

\section{REFERENCES}

[1] N. R. Anakira, "Optimal homotopy asymptotic method for solving multi-pantograph type delay differential equations," Advances in Differential Equations and Control Processes, vol. 19, no. 3, pp. 191-204, Sep. 2018, doi: 10.17654/DE019030191.

[2] N. R. Anakira, A. K. Alomari, and I. Hashim, "Optimal homotopy asymptotic method for solving delay differential equations," Mathematical Problems in Engineering, vol. 2013, pp. 1-11, 2013, doi: 10.1155/2013/498902.

[3] N. R. Anakira, A. Jameel, A.-K. Alomari, A. Saaban, M. Almahameed, and I. Hashim, "Approximate solutions of multipantograph type delay differential equations using multistage optimal homotopy asymptotic method," Journal of Mathematical and Fundamental Sciences, vol. 50, no. 3, pp. 221-232, Dec. 2018, doi: 10.5614/j.math.fund.sci.2018.50.3.1.

[4] Q. Wang and F. Fu, "Solving delay differential equations with homotopy analysis method," Life System Modeling and Intelligent Computing, pp. 144-153, 2010.

[5] R. M. Jena and S. Chakraverty, "Q-Homotopy analysis Aboodh transform method based solution of proportional delay timefractional partial differential equations," Journal of Interdisciplinary Mathematics, vol. 22, no. 6, pp. 931-950, Aug. 2019, doi: 10.1080/09720502.2019.1694742.

[6] N. R. Anakira, A. K. Alomari, and I. Hashim, “Application of optimal homotopy asymptotic method for solving linear delay differential equations," in AIP Conference Proceedings 1571, 2013, pp. 1013-1019, doi: 10.1063/1.4858786.

[7] N. R. Anakira, H. Abdelkarim, and M. Abu-Dawas, "Homotopy sumudu transformation method for solving fractional delay differential equations," General Letters in Mathematics, vol. 9, no. 1, pp. 33-41, Sep. 2020, doi: 10.31559/GLM2020.9.1.4.

[8] M. Cakir and D. Arslan, "The adomian decomposition method and the differential transform method for numerical solution of multi-pantograph delay differential equations," Applied Mathematics, vol. 06, no. 08, pp. 1332-1343, 2015, doi: 10.4236/am.2015.68126.

[9] S. Davaeifar and J. Rashidinia, "Solution of a system of delay differential equations of multi pantograph type," Journal of Taibah University for Science, vol. 11, no. 6, pp. 1141-1157, Nov. 2017, doi: 10.1016/j.jtusci.2017.03.005.

[10] M. S. M. Bahgat, "Approximate analytical solution of the linear and nonlinear multi-pantograph delay differential equations," Physica Scripta, vol. 95, no. 5, May 2020, Art. no. 055219, doi: 10.1088/1402-4896/ab6ba2.

[11] K. Jiang, "Discontinuous galerkin methods for multi-pantograph delay differential equations," Advances in Applied Mathematics and Mechanics, vol. 12, no. 1, pp. 189-211, Jun. 2020, doi: 10.4208/aamm.OA-2019-0116. 
[12] Ş. Yüzbaşı and M. Sezer, "An exponential approximation for solutions of generalized pantograph-delay differential equations," Applied Mathematical Modelling, vol. 37, no. 22, pp. 9160-9173, Nov. 2013, doi: 10.1016/j.apm.2013.04.028.

[13] M. M. Bahşi and M. Çevik, "Numerical solution of pantograph-type delay differential equations using perturbation-iteration algorithms," Journal of Applied Mathematics, vol. 2015, pp. 1-10, 2015, doi: 10.1155/2015/139821.

[14] I. Komashynska, M. Al-Smadi, A. Ateiwi, and S. Al-Obaidy, "Approximate analytical solution by residual power series method for system of fredholm integral equations," Applied Mathematics and Information Sciences, vol. 10, no. 3, pp. 975-985, May 2016, doi: 10.18576/amis/100315.

[15] M. Al-Smadi, "Solving initial value problems by residual power series method," Theoretical Mathematics and Applications, vol. 3, no. 1, pp. 199-210, 2013.

[16] H. M. Jaradat, S. Al-Shara., J. A. Khan, M. Alquran, and K. Al-Khaled, "Analytical solution of time-fractional Drinfeld-SokolovWilson system using residual power series method," IAENG International Journal of Applied Mathematics, vol. 46, no. 1, pp. 64-70, 2016.

[17] Y. Zhang, A. Kumar, S. Kumar, D. Baleanu, and X.-J. Yang, "Residual power series method for time-fractional Schrödinger equations," Journal of Nonlinear Sciences and Applications, vol. 09, no. 11, pp. 5821-5829, Nov. 2016, doi: 10.22436/jnsa.009.11.10.

[18] M. A. Bayrak, A. Demir, and E. Ozbilge, "Numerical solution of fractional diffusion equation by Chebyshev collocation method and residual power series method," Alexandria Engineering Journal, vol. 59, no. 6, pp. 4709-4717, Dec. 2020, doi: 10.1016/j.aej.2020.08.033.

[19] A. El-Ajou, M. Al-Smadi, M. N. Oqielat, S. Momani, and S. Hadid, "Smooth expansion to solve high-order linear conformable fractional PDEs via residual power series method: Applications to physical and engineering equations," Ain Shams Engineering Journal, vol. 11, no. 4, pp. 1243-1254, Dec. 2020, doi: 10.1016/j.asej.2020.03.016.

[20] M. Shqair, M. Al-Smadi, S. Momani, and E. El-Zahar, "Adaptation of conformable residual power series scheme in solving nonlinear fractional quantum mechanics problems," Applied Sciences, vol. 10, no. 3, Jan. 2020, Art. no. 890, doi: 10.3390/app10030890.

[21] I. Komashynska, M. Al-Smadi, A. Al-Habahbeh, and A. Ateiwi, "Analytical approximate solutions of systems of multipantograph delay differential equations using residual power-series method," Numerical Analysis, Nov. 2016.

[22] N. R. Anakira, "A new accurate procedure for solving nonlinear delay differential equations," Journal of Mathematical and Computational Science, vol. 11, no. 04, pp. 4673-4685, 2021.

[23] M. Syam, "A numerical solution of fractional Lienard's equation by using the residual power series method," Mathematics, vol. 6, no. 1, Dec. 2017, Art. no. 1, doi: 10.3390/math6010001.

[24] M. Al-Smadi, O. Abu Arqub, and S. Hadid, "Approximate solutions of nonlinear fractional Kundu-Eckhaus and coupled fractional massive Thirring equations emerging in quantum field theory using conformable residual power series method," Physica Scripta, vol. 95, no. 10, p. 105205, Sep. 2020, doi: 10.1088/1402-4896/abb420.

[25] B. S. Attili, "Numerical treatment of singularly perturbed two point boundary value problems exhibiting boundary layers," Communications in Nonlinear Science and Numerical Simulation, vol. 16, no. 9, pp. 3504-3511, Sep. 2011, doi: 10.1016/j.cnsns.2011.01.001.

[26] H. Allouche and A. Tazdayte, "Numerical solution of singular boundary value problems with logarithmic singularities by Padé approximation and collocation methods," Journal of Computational and Applied Mathematics, vol. 311, pp. 324-341, Feb. 2017, doi: 10.1016/j.cam.2016.08.003.

[27] S. Al-Ahmad, I. M. Sulaiman, M. Mamat, and P. Liza Ghazali, "Modified differential transform scheme for solving systems of first order ordinary differential equations," Journal of Mathematics and Computer Science, vol. 22, no. 01, pp. 73-84, Jul. 2020, doi: 10.22436/jmcs.022.01.07. 